\title{
PREFERÊNCIAS TÉRMICAS DE VACAS LEITEIRAS EM SISTEMA DE CONFINAMENTO FREESTALL
}

\author{
THERMAL PREFERENCES OF DAIRY COWS HOUSED \\ IN FREESTALL CONFINEMENT SYSTEM
}

SILVIAR. L. DE SOUZA 1

IRENILZA DE A. NÄÄS ${ }^{2}$

DANIELLA J. DE MOURA ${ }^{3}$

\section{RESUMO}

As mudanças do microclima de alojamento podem promover alterações no comportamento animal, onde fica priorizada sua preferência térmica. As medidas convencionais são pouco precisas, podendo levar a eventual erro na interpretação dos resultados. O presente trabalho teve como objetivo identificar com precisão, as preferências térmicas de vacas leiteiras alojadas em galpão de freestall, em função da ambiência do local. O experimento foi realizado em uma fazenda comercial em São Pedro, SP, onde as vacas foram monitoradas através vídeos provenientes de seis micro-câmeras coloridas com alta resolução e lente de 120 graus para registrar o local ocupado. Foram realizadas observações visuais posteriores dos vídeos, para analisar o comportamento. O galpão foi dividido em 18 quadrantes virtuais, para o mapeamento das distribuições de temperatura, umidade relativa e velocidade do ar ambientes, sendo os dados processados usando o programa computacional Surfer ${ }^{\circledR}$, para analisar a sua homogeneidade. Os resultados obtidos da análise multivariada com os dados das observações comportamentais apontaram no gráfico de Componentes Principais, as preferências das vacas consideradas importantes, por se obter resposta com vetores expressivos, quando afetadas pelo ambiente de alojamento: bebendo água, comendo e deitadas na cama. As distribuições do ambiente dentro do galpão não foram homogêneas e variavam em função do uso dos equipamentos de climatização. Concluiu-se que as vacas tiveram, prioritariamente, preferência pelos locais com ambiente mais ameno e velocidade de ar mais alta.

Palavras-chave: Ocupação física, Componentes Principais, zootecnia de precisão

\section{ABSTRACT}

The differences in the rearing microclimate may promote changes in the animal behavior, when its thermal preference of higher priority is shown. Conventional measurements are not precise leading to eventual error in the results interpretation. This present research

\footnotetext{
${ }^{1}$ Bolsista Fapesp. Faculdade de Engenharia Agrícola (FEAGRI) - UNICAMP, Campinas, SP, e-mail: silucas_souza@hotmail.com

${ }^{2}$ Professor Titular Voluntária. Faculdade de Engenharia Agrícola (FEAGRI), UNICAMP, Campinas, SP.

${ }^{3}$ Professor Doutor. Faculdade de Engenharia Agrícola (FEAGRI) - UNICAMP. Cx. P. 6011. 13083-970, Campinas, SP.
} 
had the objective to identify with precision the thermal preferences of dairy cows reared in freestall barn, as function of the local environment. The experiment was conduced in a commercial dairy farm located at São Pedro, SP, where the cows were monitored through videos taken with six colored high resolution video cameras, and 120 degrees lens for registering the local occupancy. Visual observations were made afterwards using the videos for analyzing the behavior. The barn was divided into 18 virtual quadrants for the mapping of the ambient temperature, relative humidity and air velocity distribution, and the data were processed using the software Surfer ${ }^{\circledR}$ for analyzing its homogeneity. The results from the multivariable analysis using the observed behavioral data showed in the graph of Main Components the cows preference considered important for obtaining an expressive vector answer when affected by the rearing environment: drinking water, eating, and laying down on bedding. The environmental distribution inside the barn was not homogeneous, and varied as function of the use of acclimatization equipments. It was concluded that the cows had priority preference by the places where the environment is milder, and the air speed is higher.

Keywords: Physical occupancy, Principal Components, Precision Animal Production

\section{INTRODUÇÃO}

A vaca é um animal homeotermo, com temperatura corporal entre $38,4-39^{\circ} \mathrm{C}$, mantida com o equilíbrio entre o calor produzido e/ou recebido e sua dissipação para o ambiente (ALBRIGHT, 1993; NIENABER et al., 2003; BERMAN, 2005). Quando a temperatura corporal se eleva podem ocorrer eventos, tais como a evaporação da umidade através da superfície do corpo (sudação) e pelos pulmões (PIRES et al., 2001; BERMAN, 2005; SMITH et al., 2006). O animal exposto a extremos de temperaturas, experimenta o estresse, seja por excesso de frio ou calor (ARCARO, 2000; WEST, 2003) aumentando a circulação periférica e diminuindo a circulação nos órgãos internos. Segundo CAMPOS et al. (2000), as perdas na produção de leite, quando o animal se encontra em estresse térmico, podem atingir uma queda de até $20 \%$, em comparação com rebanhos alojados em condições ambientais de conforto térmico.

O comportamento animal revela a ligação entre o organismo, o sistema nervoso e o ecossistema, e ainda representa a parte de um organismo, através da qual há a interação com o ambiente (SNOWDON, 1999; SARIEGO,
2005). Em períodos quentes do dia, as vacas geralmente preferem deitar-se em corredores molhados, a estar nas camas (FRAZZI et al., 2000), provavelmente para se beneficiarem do resfriamento evaporativo. Os animais diminuem as atividades nas horas mais quentes do dia, permanecendo deitados na área de descanso (BLACKSHAW \& BLACKSHAW, 1994). DAMASCENO et al. (1999) determinaram que, o padrão de idas ao comedouro, é relacionado à preferência térmica pelas vacas ao ambiente. A observação visual do comportamento ingestivo, de ruminação, ócio e consumo de água de bovinos, foram usadas com sucesso por DAMASCENO et al. (1999), QUEIROZ et al. (2001) e TREVISAN et al. (2004). Novas técnicas de interpretação de dados permitem uma análise qualitativa na interpretação da interação animal/ambiente, dentre elas, a Análise de Componentes Principais é usada para identificar possíveis associações entre variáveis de um conjunto de dados (JOHNSON \& WICHERN, 1998). Essa técnica foi usada por LALONI et al. (2004), PEREIRA (2005) e SALGADO (2006), para o entendimento da correlação entre a ambiência e as variáveis produtivas, bem como as expressões corporais dos animais. Este trabalho visou identificar a 
preferência térmica de vacas leiteiras da raça Holandesa, em termos de uso do espaço físico ocupado, em relação ao ambiente do alojamento.

\section{MATERIAL E MÉTODOS}

Foram utilizadas 80 vacas oriundas de um rebanho comercial com características homogêneas (multíparas em lactação, peso médio $600 \mathrm{~kg}$ ) da raça Holandesa puro por cruza, estando em fase de lactação semelhante, entre 90-100 dias de lactação, com uma produção média de $30 \mathrm{~kg}$ de leite/ dia. As vacas eram ordenhadas três vezes ao dia (01h:00min, 09h:00min e 17h:00min.). Os horários de arraçoamento (07h:00min. e 15h:00min) e a dieta alimentar foram mantidos conforme a rotina da fazenda. A dieta consistiu em silagem da gramínea Tifton, polpa cítrica, silagem de milho, milho úmido, silagem de alfafa pré-secada, caroço de algodão e concentrado.

A instalação do tipo freestall era localizada na região de São Pedro, estado de São Paulo, com latitude $22^{\circ} 32^{\prime} 55^{\prime \prime}$, longitude $47^{\circ} 54^{\prime} 50^{\prime \prime} \mathrm{O}$ e altitude de $580 \mathrm{~m}$. O clima local é Cwa da classificação Köppen, quente e úmido com estação chuvosa no verão e seco no inverno. As médias anuais de temperatura e pluviometria eram de $22^{\circ} \mathrm{C}$ e $1.200 \mathrm{~mm}$, respectivamente, com ventos predominantes SE. As dimensões do galpão eram de $28 \times 80 \mathrm{~m}$, com cumeeira de $9 \mathrm{~m}$ e pé direito de $3,5 \mathrm{~m}$, com capacidade para alojar 200 vacas, em dois lotes. A instalação era totalmente aberta, com pilares de madeira e o piso de concreto com ranhuras, tendo um corredor central medindo $2,90 \mathrm{~m}$ de largura. As camas, com dimensões de 1,10x2,12m (114 em duas fileiras de 57), eram forradas com sacos de tecido impermeável recheados com borracha de pneu e pó de serra,

Para o mapeamento das condições termo-higrométricas, foi selecionada a área mais visitada pelos animais na área central do galpão, medindo $24,0 \mathrm{~m}$ de comprimento por 12,0m de largura. Esta área foi dividida em 18 quadrantes virtuais (Figura 1), distantes $4,0 \mathrm{~m}$ cada um e os dados do ambiente de temperatura externa (Text, $\left.{ }^{\circ} \mathrm{C}\right)$, mínima $\left(\mathrm{Tmin},{ }^{\circ} \mathrm{C}\right)$ e máximas $\left(\mathrm{Tmáx},{ }^{\circ} \mathrm{C}\right)$, umidade relativa média (UR,\%) e velocidade do ar (Var,m/s) foram registradas no centro geométrico de cada quadrante, sendo utilizado o mesmo planejamento esquemático na ordem de coordenadas $X$ e Y, para o mapeamento nos quadrantes.
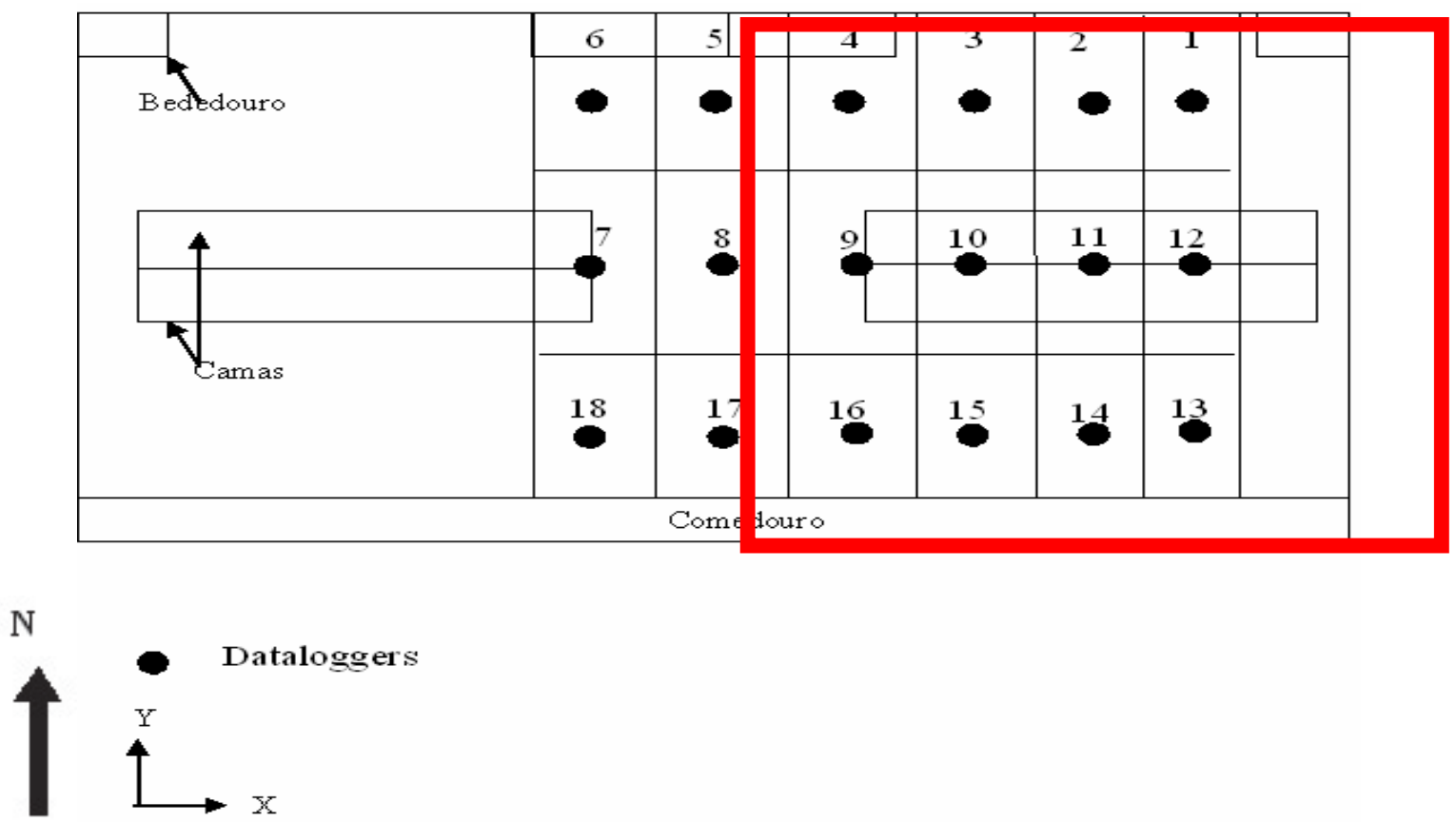

Figura 1. Esquema da área e dos centros geométricos dos quadrantes e fixação dos registradores eletrônicos de temperatura e umidade relativa. 
Para o registro de Text, Tmáx, Tmin e UR foram utilizados 18 dataloggers da marca $\mathrm{HOBO}^{\circledR}$, programados para a coleta de dados a cada $15 \mathrm{~min}$, de 25 a 27 de setembro de 2006, a uma altura de $2,5 \mathrm{~m}$ do piso. A Var foi coletada usando um anemômetro digital Kastel ${ }^{\circledR}$, colocado no centro de cada quadrante, usando a mesma freqüência e o mesmo horário das demais variáveis. Com os dados de Tmáx e Tmin, foi calculada a temperatura média $\left(\mathrm{Tm},{ }^{\circ} \mathrm{C}\right)$ de cada quadrante.

O programa computacional SURFER ${ }^{\circledR}$ (1999) foi utilizado para se obter um mapa de isolinhas dos dados médios (Tm, Ur e Var) dos dias, nos quadrantes, por meio da inserção dos pontos onde foram coletados $\left(\mathrm{X}_{\mathrm{i}}, \mathrm{Y}_{\mathrm{i}}\right)$ os dados das condições climáticas na área dos quadrantes, em cada dia.

Foram realizadas observações visuais prévias dos locais mais visitados pelas vacas durante o dia, à distância, durante uma hora. Também foram consultados os tratadores, para entendimento do comportamento das vacas, em todos os horários, de maneira a se conhecer previamente, o local de sua preferência. Quanto à ocupação física de cada área, as vacas foram observadas, de acordo com as informações constantes na Tabela 1.

Tabela 1. Descrição da ocupação física pelas vacas, em áreas específicas do galpão de alojamento freestall.

O registro da ocupação física dos locais (comedouro, bebedouro e cama) foi realizada utilizando um sistema de captura de imagens, composto por seis microcâmeras RGB (36mm x 36mm), analógicas, de 420 linhas horizontais de resolução, sensibilidade mínima de $1 \mathrm{Lux}, 12 \mathrm{~V}$ de tensão à $120 \mathrm{~mA}$, e com lente convergente de 3,6mm. As câmeras, com lentes de 120 graus, foram instaladas no galpão a uma altura de cinco metros do piso, em ângulo apropriado, de forma a obter a imagem mais precisa das vacas.

A observação das vacas foi feita através da visualização dos vídeos, utilizando o método adotado por OVERTON et al. (2003), FRAZZI et al. (2000), GAWORSKI et al.(2003) e MÜLLER \& SCHRADER, 2005), por um período de 10min para cada observação, para subsidiar a análise da ocupação dos locais, pelas vacas leiteiras. As imagens foram observadas em seis vídeos distintos, em dois períodos, em torno das 07h:00min e das $15 \mathrm{~h}: 30 \mathrm{~min}$, por 10min, denominandose esta obsersação de "visual espaçada". O percentual de distribuição foi obtido através do número de vacas que permaneciam majoritariamente num determinado local e pelo número total de vacas presentes na filmagem.

Para a análise da preferência térmica, a informação do local ocupado foi tabulada, bem como o todo o registro termohigrográfico da área dos quadrantes, de maneira a permitir a interação dos dados do ambiente térmico e da preferência das 
vacas por determinados locais. Para a análise multivariada, utilizou-se o método de gráficos de Componentes Principais, sendo os dados processados usando o programa computacional MINITAB ${ }^{\circledR}$ (2004).

\section{RESULTADOS E DISCUSSÃO}

Não houve diferença significativa entre os valores de temperatura externa e interna $(\mathrm{P}<0,001)$. A Figura 2 mostra os mapas da distribuição espacial das temperaturas médias nos quadrantes nos dia 25, 26 e 27 , respectivamente. Os tons em azul representam as temperaturas mínimas e as máximas são representadas em tons de vermelho dentro do galpão.

(a)

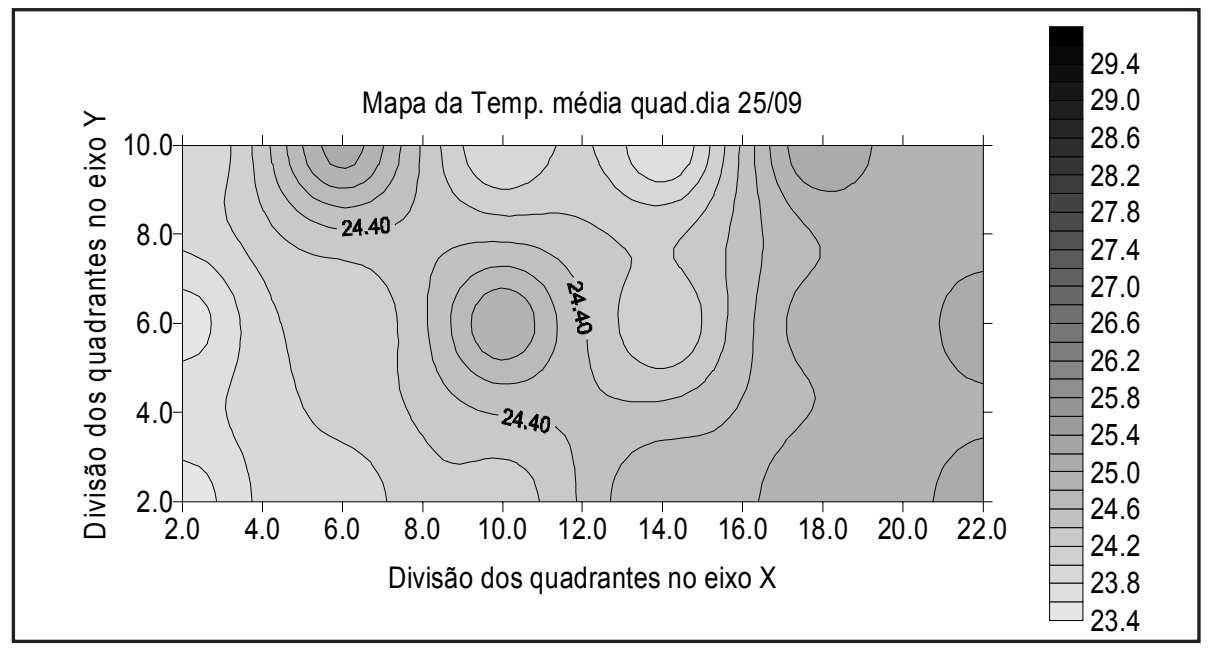

(b)

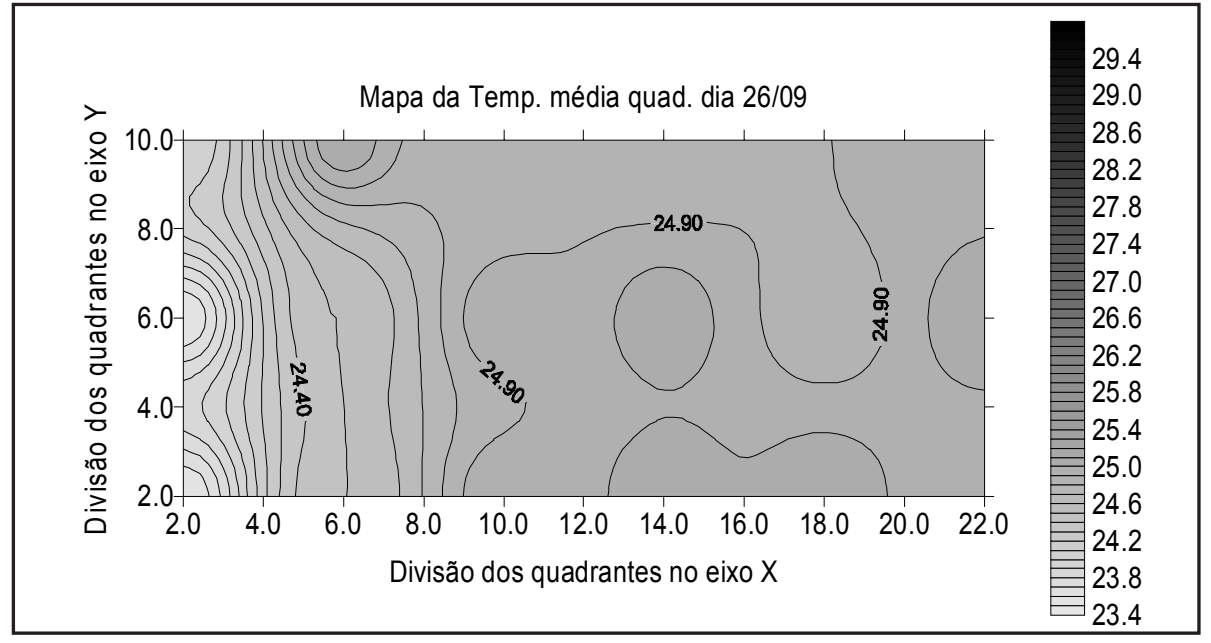

(c)

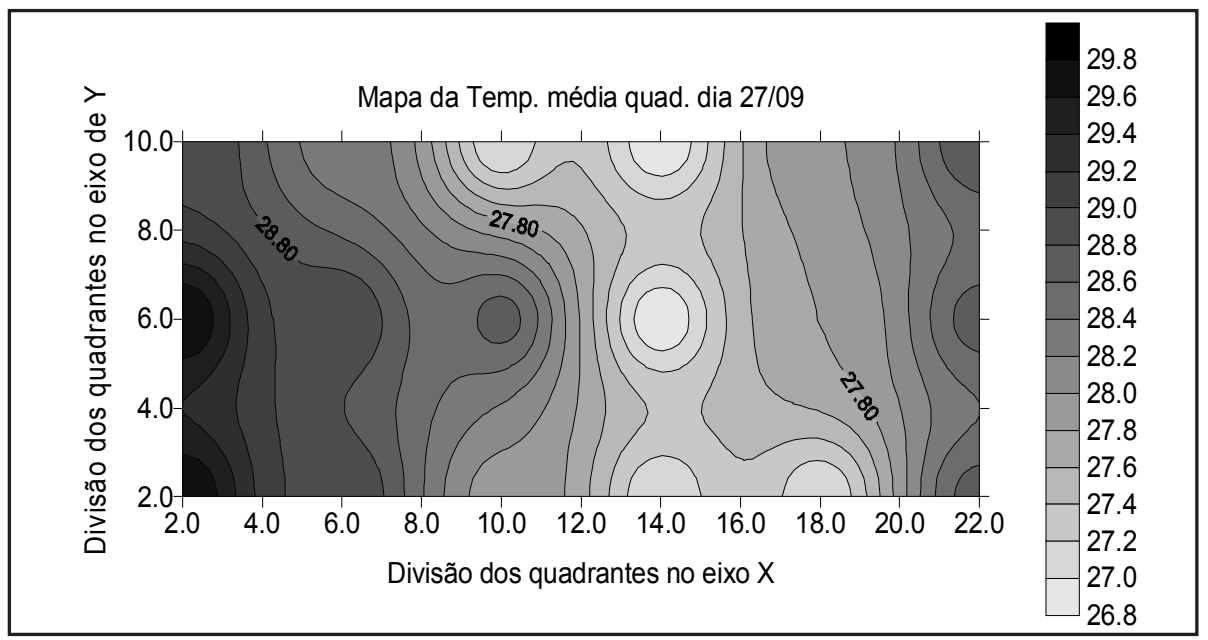

Figura 2. Mapeamento da espacialização da temperatura média diária nos quadrantes virtuais. 
Nas Figuras 2a e 2b as temperaturas não sofreram grandes variações $(23,4$ $25,0^{\circ} \mathrm{C}$ ), mostrando certa homogeneidade no ambiente térmico nestes dias. A Figura 2c, mostra a incidência de temperaturas maiores nos quadrantes $5,6,7,8,9,17$ e 18 , com valores variando de $26,8-28,8^{\circ} \mathrm{C}$, enquanto nos demais quadrantes foram menores, uma vez que, nestes quadrantes havia ventiladores e aspersores que eram acionados automaticamente, quando a temperatura atingia $25^{\circ} \mathrm{C}$. Já nas regiões dos comedouros e camas, houve homogeneidade nos quadrantes, com temperaturas de $28-29^{\circ} \mathrm{C}$. A literatura é controversa quanto às temperaturas consideradas ideais para o conforto térmico de bovinos leiteiros, que vão de 4 a $26^{\circ} \mathrm{C}$, em função da raça, idade e metabolismo do animal e, NIENABER et al. (2003) afirmam que, o comportamento de rebanho bovino é afetado pelo estresse térmico em temperaturas em torno de $30^{\circ} \mathrm{C}$, o que não aconteceu durante os três dias consecutivos.

Não se procedeu com o mapeamento da UR média nos mesmos quadrantes acima citados, pois houve homogeneidade entre os locais $(P>0,05)$, não influenciando na preferência térmica dos animais pela Análise das Componentes Principais.

A Figura 3 mostra o mapeamento da Var média nos quadrantes. Na Figura 3a e 3 b nota-se uma baixa variação na Var. $\mathrm{Na}$ Figura 3c observou-se que as Var médias do ar nos quadrantes $4,9,10,15$ e 18 foram mais baixas $(0,4-0,6 \mathrm{~m} / \mathrm{s})$ que nos demais quadrantes, que variaram entre $0,6-0,7 \mathrm{~m} / \mathrm{s}$, influenciadas pelo uso dos ventiladores associados a aspersores, que se encontravam ligados $\left(\mathrm{Tm}>25^{\circ} \mathrm{C}\right)$. 
(a)

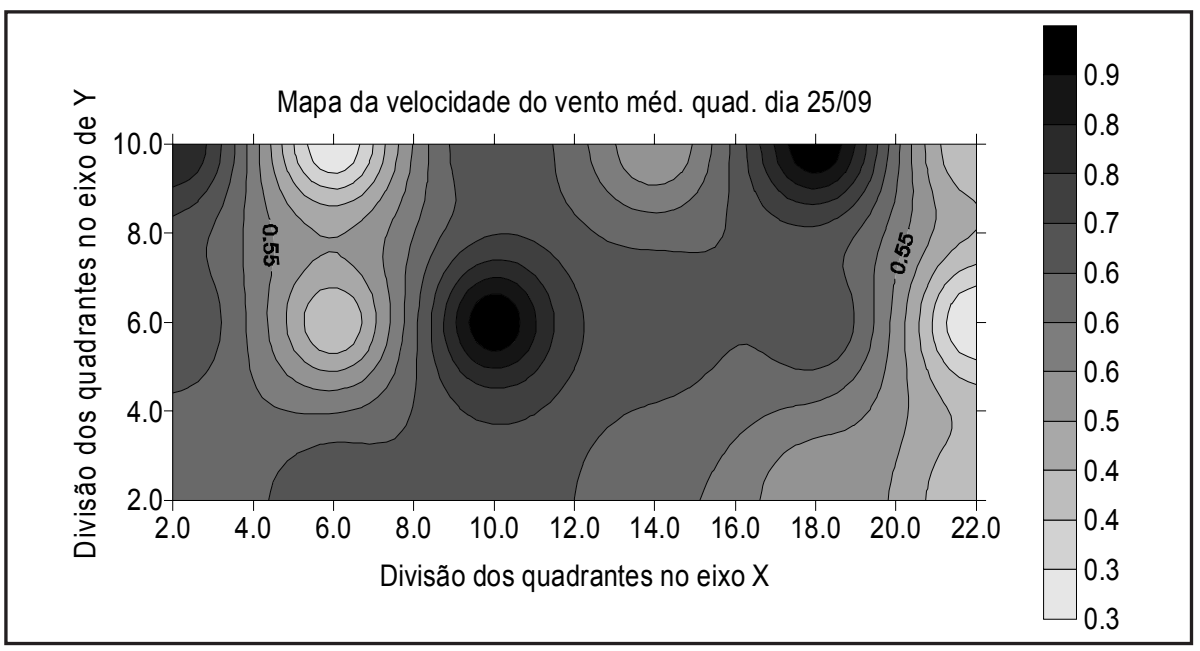

(b)

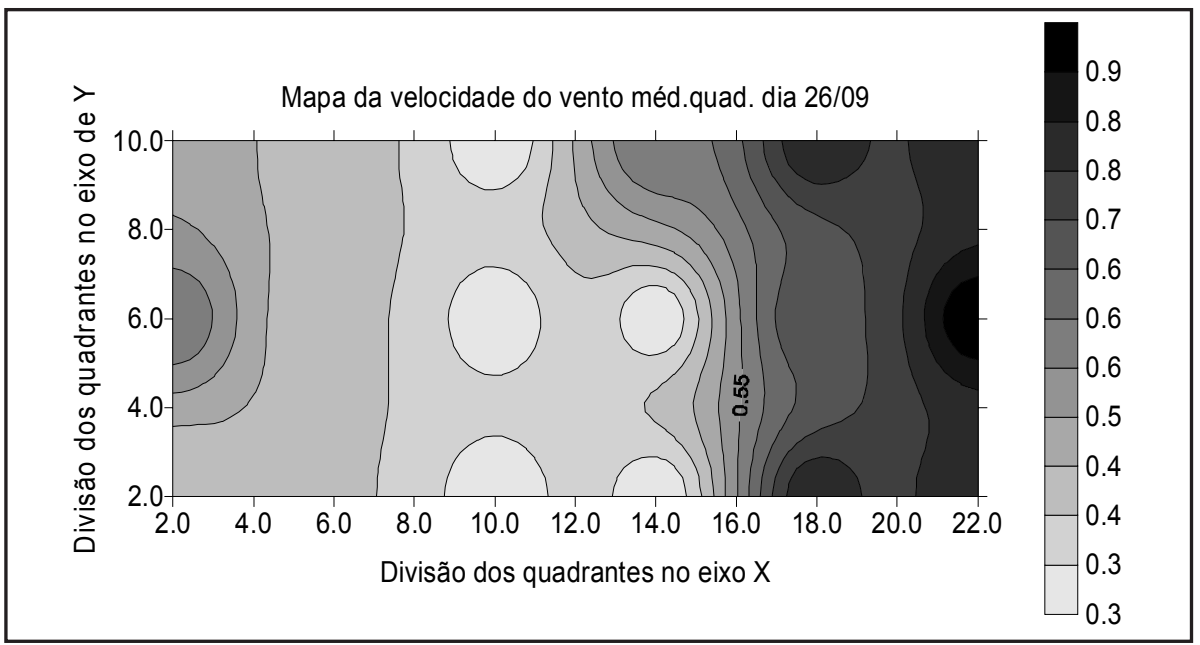

(c)

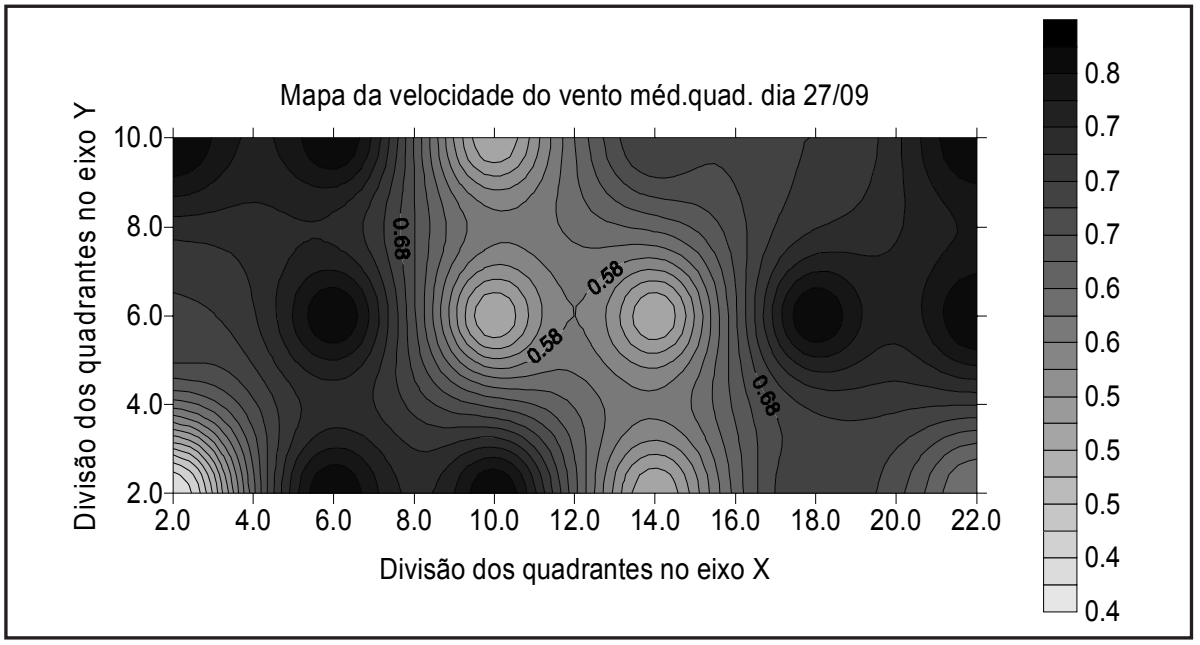

Figura 3. Mapeamento espacialização da velocidade média do ar diária nos quadrantes virtuais.

O efeito do sistema de resfriamento adiabático, associado à ventilação forçada, diminui a temperatura de pele, melhorando a sensação térmica do animal (BROUK et al., 2003). Aexigência de ventilação em uma instalação, segundo BACCARI (2001) e KADZERE et al.(2001) obedece a critérios distintos, dependendo de sua finalidade (higiene ou conforto térmico) e, conhecendose as relações funcionais entre o animal e o ambiente, pode-se chegar a maior eficiência na produção (DAMASCENO et al., 1999). O uso dos ventiladores pode, portanto, ter interferido na preferência das vacas pelos locais onde havia Var era mais alta, em dias mais quentes. 
Quanto à observação da ocupação espacial, a Tabela 2 mostra a percentagem do tempo que as vacas ocupavam os locais estudados.

Tabela 2. Percentagem de vacas presentes nos locais de sua preferência, através da visualização de seis vídeos.

\begin{tabular}{llllll}
\hline \multicolumn{6}{l}{ Locais ocupados pelas vacas } \\
Dia/Mês Horário (h:min) & Vídeo & Bebedouro (\%) & Comedouro (\%) & Cama (\%) \\
\hline 25/set & $07: 00$ & 1 & 2,3 & 1,2 & 4,6 \\
25/set & $15: 30$ & 2 & 5,8 & 2,3 & 1,2 \\
26/set & $07: 00$ & 3 & 5,8 & 2,3 & 7,0 \\
26/set & $15: 30$ & 4 & 3,5 & 1,2 & 7,0 \\
27/set & $07: 00$ & 5 & 3,5 & 1,2 & 5,8 \\
27/set & $15: 30$ & 6 & 9,3 & 1,2 & 7,0 \\
\hline
\end{tabular}

O emprego da técnica de Análise de Componentes Principais (Figura 4) mostrou as correlações encontradas entre os valo- res do ambiente térmico e os locais ocupados pelas vacas.

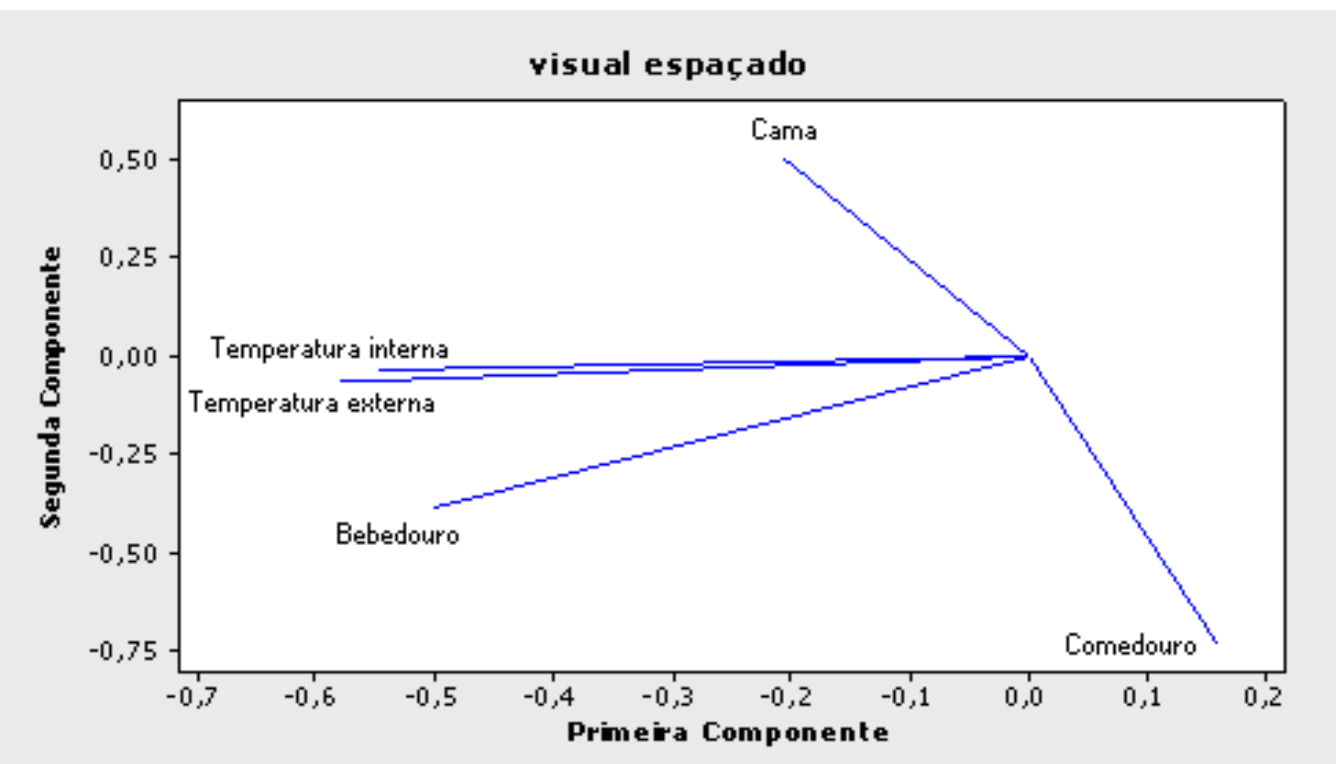

Figura 4. Gráfico de Componentes Principais indicando os vetores das preferências de uso dos locais estudados, através da análise visual em períodos espaçados (07h:00min e 15h:30min), em função dos dados de ambiente.

Em primeiro lugar observou-se a magnitude dos vetores e o posicionamento relativo entre eles. Os vetores das temperaturas externas e internas mostraram direção e sentido próximos, estando fortemente associados positivamente e foram correlacionadas com a atividade de beber água, que esteve associada negativamente com a utilização do comedouro. Resultados semelhantes foram encontrados por HUBER (1990), ALBRIGHT (1993), PIRES et al. (2000), CAMPOS
(2000) e COSTA \& BROOM (2001). Como as temperaturas altas levam a maior ingestão de água, a preferência pelo bebedouro central pode ser explicada pela sua dimensão (maior que os laterais), reduzindo a competição por espaço. Resultados semelhantes são citados por MACHADO FILHO et al. (2004) e TEIXEIRA et al. (2006).

A freqüência de utilização do comedouro foi inversamente associada com a utilização da cama e, como acima das 
camas existiam ventiladores e aspersores, que eram ligados sempre que a temperatura atingia $25^{\circ} \mathrm{C}$, as vacas procuravam sua preferência térmica em função do aumento da Var (FRAZZI et al, . 2000; CALEGARI et al., 2003 e PERISSINOTO, 2006). A freqüência de alimentação associou-se negativamente com o aquecimento do ambiente interno e externo, como era de se esperar.

Como os dados foram coletados nos períodos de incidência de Tmin e Tmáx (07h:00 min e 15h:30min) as respostas comportamentais foram mais claramente visualizadas. Nem a UR média, ou a Var média se mostraram significativas no gráfico das Componentes Principais. Segundo o gráfico, quanto maior a freqüência de uso da cama e do comedouro, menor o uso do bebedouro, por não serem correlatas. Isto pode ser justificado pelo fato de, apesar das temperaturas não apresentassem valor alto suficiente para ser considerado um estado de estresse térmico, as vacas preferiram os locais onde havia ventiladores e nebulizadores.

\section{REFERÊNCIAS BIBLIOGRÁFICAS}

ALBRIGHT, J.Feeding behavior of dairy cattle, Journal of Dairy Science, Savoy, IL. v.76, p.485-498, 1993.

ARCARO, I, J. Avaliação da influência de ventilação e aspersão em coberturas de sombrite para vacas em lactação. Campinas. 2000. 81p. Tese (Doutorado) Universidade Estadual de Campinas.

BACCARI JR., F. Manejo ambiental da vaca leiteira em climas quentes. Londrina: UEL, 2001. 142p.

BERMAN, A. Estimates of heat stress relief needs for Holstein dairy cows. Journal of Animal Science, v. 83, n.6, p.1377-1384, 2005.
No local da cama houve maior Var (quadrantes 10, 11e 12), que foram de 0,5$0,8 \mathrm{~m} / \mathrm{s}$, o que induziu à preferência das vacas, como preconizam TURNER et al. (1992), FRAZZI et al. (2000) e PERISSIONOTO (2006).

\section{CONCLUSÕES}

A preferência térmica das vacas foi por locais onde havia ambiente mais ameno ou maior movimentação e ar. Houve alta correlação entre a preferência de uso do bebedouro ingerindo água, quando as temperaturas estiveram altas. A correlação entre a temperatura ambiente e uso do comedouro foi negativa.

\section{AGRADECIMENTOS}

Os autores agradecem à Embrapa (PRODETAB n. 056-02), a Douglas D'Alessandro Salgado, à Fazenda Campestre e à FAPESP.
BLACKSHAW, J. K.; BLACKSHAW, A. W. Heat stress in cattle and the effect of shade on production and behaviour: a review. Australian Journal of Experimental Agriculture, v. 34, p. 285-295, 1994.

BROUK, M. J.; SMITH, J. F.; HARNER III, J. $P$. Effect sprinkling frequency and air flow on respiration rate, body surface temperature and body temperature of heat stressed dairy cattle. In: INTERNATIONAL DAIRY HOUSING CONFERENCE, 5. Fort Worth, 2003. Proceedings. Fort Worth: American Society of Agricultural Engineers, 2003. p. 263-268.

CALEGARI, F.;CALAMARI, L.; FRAZZI, E. Effects of ventilation and misting on behaviour of dairy cattle in the hot season in 
South Italy. In: INTERNATIONAL DAIRY HOUSING CONFERENCE, 5. Fort Worth, 2003. Proceedings. Fort Worth: American Society of Agricultural Engineers, 2003. p. 303-311.

CAMPOS A. T. A importância da água para o gado de leite. (on-line). Juiz de Fora: Embrapa Gado de Leite. 2000. Disponível em : < http://www.sbz.org.br >. Acesso em 20 out 2006.

COSTA, M. J. R.; BROOM, D. M.Consistency of side choice in the milking parlour by Houstein-Friesian cows and its relationship with their reactivity and milk yeld. Applied Animal Behaviour Science, v.70, p.177-186. 2001.

DAMASCENO, J.C; BACCARI JR., F.; TARGA, L.A. Respostas comportamentais de vacas holandesas, com acesso a sombra constante ou limitada. Pesquisa Agropecuária Brasileira, Brasília, v. 34, n.4, p. 709-715, 1999.

FRAZZI, E.; CALAMARI, L.; CALEGARI, F.; STEFANINI, L. Behavior of dairy cows in response to different barn cooling systems. Transactions of the American Society of Agricultural Engineers, Michigan, v. 43, n.2, p. 387-394, 2000.

GAWORSKI, M.A.; TUCKER, C. B.; WEARY, D. M.; SWIFT, M. L. Effects of stall design on dairy cattle behaviour. In: INTERNATIONAL DAIRY HOUSING, 5. Fort Worth, 2003. Proceedings. Fort Worth:, American Society of Agricultural Engineers, 2003, p.139-146.

HUBER, J. T. Alimentação de vacas de alta produção sob condições de stress térmico. In: ENCONTRO DE BOVINOCULTURA LEITEIRA,1., Piracicaba. Anais... Piracicaba: FEALQ, 1990.p. 33-48.

JOHNSON, R.A.; WICHERN, D.W.Applied multivariate statistical analysis. Rio de Janeiro:RG Editora Prentice-Hall do Brasil, 1988. 816p.
KADZERE. C. T.; MURPHY, M. R.; SILANIKOVE, N.; MALTZ, E. Heat stress in lactating dairy cows: a review. Livestock Production Science, v.77, p 59-91, 2001.

LALONI, L. A.; NÃ̃̃S, L. A.; MACARI, M.; PEREIRA, D.F.; PINHEIRO, M.G. Índice de Previsão de produção de leite para vacas Jersey. Engenharia Agrícola, Jaboticabal, SP, v. 24, n.2, p.246-254, 2004.

MACHADO FILHO, L.C.P.; TEIXEIRA, D.L.; WEARY, D.M.; KEYSERLINGK,M.A.G. von $g$ better water troughs: dairy cows prefer and drink more from larger troughs. Applied Animal Behaviour Science, v. 89, p.185193, 2004.

MACHADO FILHO, L.C.P.; TEIXEIRA, D.L.; WEARY, D.M.; KEYSERLINGK, M.A.G. von; HÖTZEL. M J. Designing better water troughs: dairy cows prefer and drink more from larger troughs. Applied Animal Behaviour Science, v. 89, p.185-193, 2004.

MINITAB $^{\circledR}$ : Minitab Statistical Software for Windows. São Paulo: Award Brasil, 2004. Disponível em <http://www.minitab.com/ products/minitab $>$. Acesso em 10 de fevereiro de 2004.

MÜLLER, R.; SCHRADER, L. A new method to measure behavioural activity levels in dairy cows. Applied Animal Behaviour Science, v.83, p. 247-258, 2003.

NIENABER, J.A.; HAHN, G.L.; BROWNBRANDI, T.M.; EIGENBERG, R.A. Heat stress climatic conditions and the physiological responses of cattle. In: INTERNATIONAL DAIRY HOUSING, 5, Fort Worth, 2003. Proceedings... Fort Worth:, American Society of Agricultural Engineers, 2003.p. 255-262.

OVERTON, M. W.; SISCHO, W. M.; DECHANT, G.; MOORE, D.A. Observations of dairy cattle behavior using time-lapse photography in a California free-stall barn. In: ANNUAL CONFERENCE AMERICAN 
ASSOCIATION OF BOVINE PRACTITIONERS, 33. 2000, Stillwater. Proceedings... Stillwater: AABP, 2000. p.38-139.

PERISSINOTTO, M.; MOURA, D.J.; MATARAZZO, S.V.; MENDES, A.S.; NÃÃS, I.A. Behavior of dairy cows housed in environmentally controlled freestall. Agriculture Engineering International CIGR E-Journal, v. 8, n.1, p.1-11, 2006.

PEREIRA, D. F. Metodologia para estimativa de bem-estar de matrizes de frango de corte utilizando monitoramento digital e construção de modelos de simulação. 2005. 155p. Tese (Doutorado) - Universidade Estadual de Campinas.

PIRES, M. F. A.; TEODORO, R. L.; CAMPOS, A. T. Efeitos do estresse térmico sobre a produção de bovinos. In: CONGRESSO NORDESTINO DE PRODUÇÃO ANIMAL, 8., 2000, Teresina. Anais... Teresina, PI: SNPA, 2000. p. 87104.

PIRES, M. F.A.; VERNEQUE, R. S.; VILELA, D. Ambiente e comportamento na produção de leite. Revista Informe Agropecuário, Belo Horizonte , MG, v. 22, n. 211, p11-21, 2001.

QUEIROZ, A. C.; NEVES, J. S. MIRANDA, J.C.; PEREIRA,E.S. DUTRA, A.R. Efeito do nível de fibra e da fonte de proteína sobre o comportamento alimentar de novilhas mestiças Holandês-Zebu. Arquivo Brasileiro de Medicina Veterinária e Zootecnia, Belo Horizonte, MG, v. 53, n. 1, p. 84-88, 2001.

SALGADO, D. A. Modelo estatístico para predição de bem-estar de reprodutoras de frango de corte baseado em dados de ambiente e análise do comportamento. Campinas, 2006. 126p. Dissertação (Mestrado) - Universidade Estadual de Campinas.
SARIEGO, J. C. L. O que é Etologia? São Paulo. Editora Scipione, 2003. Disponível em <http://paginas.terra.com.br/educacao/ $\begin{array}{llllllll} & a & r & i & e & g & 0 & l\end{array}$ o_estudo_do_comportamento.htm>. Acesso em 29 de abril de 2005.

SMITH, T.R.; CHAPA, A.; WILLARD, S.; HERNDON JR. C.; WILLIANS, R. J.; CROUCH, J.; RILEY, T.; POGUE, D. Evaporative tunnel cooling of dairy cows in the southeast. I: effect on body temperature and respiration rate. Journal of Dairy Science, Savoy, II. v. 89, n.10, p. 3904-3914, 2006.

SNOWDON, C. T. O significado da pesquisa em comportamento animal. Estudos de Psicologia, Natal, RN, v.4, n.2, p. 365-373, 1999.

SURFER囚.: Golden Software. Colorado, U.S.A.: Inc.Golden 1997. Disponível em: <http://www.goldensoftware.com>.

TEIXEIRA, D. L.; HÖTZEL, M. J.; MACHADO FILHO, L. C. Designing better water troughs: 2. Surface area and height, but not depth, influence dairy cows' preference. Applied Animal Behaviour Science, v.96, n.1-2, p. 169-175, 2006.

TREVISAN, N.B.; QUADROS, F.L. F.; SILVA, A.C. F.; BANDINELLI, D.G.; MARTINS, C.E.N.; SIMÕES, L.F.C.; MAIXNER, A.R.; PIRES, D.R.F. Comportamento ingestivo de novilhos de corte em pastagem de aveia preta e azevém com níveis distintos de folhas verdes. Ciência Rural, Santa Maria, RS, v. 34, n.5, p. 1543-1548, 2004.

TURNER, L. W.; CHASTAIN, J. P.; HEMKEN, R.W.; GATE, S. R. S. Reducing heat stress in dairy cows through sprinkler and fan cooling. Applied Engineering in Agriculture, v.8, n. 3, p. 375-379, 1992.

WEST, J. W. Effects of heat-stress on production in dairy cattle. Journal of Dairy Science, Savoy, IL, v. 86. 